\title{
Persistence of bactericidal antibodies following early infant vaccination with a serogroup $B$ meningococcal vaccine and immunogenicity of a preschool booster dose
}

\author{
Matthew D. Snape MBBS MD, Praveen Saroey MBBS, Tessa M. John BSc(Hons), Hannah Robinson DipHE, \\ Sarah Kelly MSc, Nicoletta Gossger MD, Ly-Mee Yu MSc, Huajun Wang MSc, Daniela Toneatto MD, \\ Peter M. Dull MD, Andrew J. Pollard MBBS PhD
}

\begin{abstract}
Background: The multicomponent serogroup B meningococcal (4CMenB) vaccine was recently licensed for use in Europe. There are currently no data on the persistence of bactericidal antibodies induced by use of this vaccine in infants. Our objective was to evaluate serogroup B-specific bactericidal antibodies in children aged 40-44 months previously vaccinated at 2, 4, 6 and 12 months of age.
\end{abstract}

Methods: Participants given 4 doses of $4 C M e n B$ as infants received a fifth dose of the vaccine at 40-44 months of age. Age-matched participants who were MenB vaccine-naive received $4 C M e n B$ and formed the control group. We evaluated human complement serum bactericidal activity (hSBA) titres at baseline and 1 month after each dose of 4 CMenB.

Results: Before a booster dose at enrolment, $41 \%-76 \%$ of 17 participants previously vaccinated with 4 CMenB in infancy had hSBA titres of 4 or greater against 4 reference strains. Before vaccination in the control group ( $n=$ 40) these proportions were similar for strains 44/76-SL (63\%) and M10713 (68\%) but low for strains NZ98/254 (0\%) and 5/99 (3\%). A booster dose in the 4 CMenB-primed participants generated greater increases in hSBA titres than in controls.

Interpretation: As has been observed with other meningococcal vaccines, bactericidal antibodies waned after vaccination with 4CMenB administered according to an approved infant vaccination schedule of 2, 4, 6 and 12 months of age, but there was an anamnestic response to a booster dose at 4044 months of age. If 4 CMenB were introduced into routine vaccination schedules, assessment of the need for a booster dose would require data on the impact of these declining titres on vaccine effectiveness. ClinicalTrials.gov, no. NCT01027351
A vaccine against serogroup B meningococcus has recently been licensed for use in Europe ${ }^{1}$ and is being considered for licensure in Canada. This vaccine, known as multicomponent serogroup B meningococcal (4CMenB) vaccine, consists of 3 recombinant proteins: factor $\mathrm{H}$ binding protein (fHbp), Neisseria adhesin A (NadA) and Neisseria heparin binding antigen (NHBA) combined with detoxified outer membrane vesicles from the strain responsible for an epidemic of serogroup B meningococcal disease in New Zealand (NZ98/254). Clinical trials of 4CMenB have shown it to be immunogenic against reference strains selected to specifically express one of the vaccine antigens. ${ }^{2-6}$ On the basis of these trials, the approved schedule for infants aged 2 to 5 months is 3 doses given at least 1 month apart, with a booster dose given at 12 to 23 months of age. ${ }^{7}$ The persistence of vaccine- induced antibodies throughout childhood following this booster dose is unknown, but it is particularly relevant because the incidence of invasive serogroup B meningococcal disease in children aged 1 to 4 years is second only to the incidence in children less than 1 year of age. ${ }^{8}$

In this study, we assessed the persistence of these bactericidal antibodies in children aged 40 44 months who had previously received either $4 \mathrm{CMenB}$ or a vaccine containing the recombinant proteins alone (recombinant protein serogroup B meningococcal [rMenB] vaccine) at 2, 4, 6 and 12 months of age. ${ }^{3}$ We also assessed the immunogenicity and reactogenicity of a booster dose.

\section{Methods}

\section{Participants}

Participants in the original phase II study were recruited at 2 sites: 12 at the Gloucester Vaccine
Competing interests: See end of article for competing interests.

This article has been peer reviewed.

Correspondence to:

Matthew Snape,

matthew.snape@paediatrics .ox.ac.uk

CMAJ 2013. DOI:10.1503 /cmaj.130257 
Evaluation Unit and 135 at the Oxford Vaccine Group, University of Oxford. In the original study, infants were randomly assigned 2:2:1:1 to receive $4 \mathrm{CMenB}$ at $2,4,6$ and 12 months; rMenB at 2, 4, 6 and 12 months; 4 CMenB at 12 months; or rMenB at 12 months (Figure 1). All 125 participants who completed the original study at the Oxford site were invited to take part in this follow-up study, and we planned to recruit $50 \mathrm{MenB}$ vaccine-naive, age-matched participants as controls.

Inclusion criteria were healthy children aged 40-44 months who had completed the original study, or, for controls, children of this age who had not previously received a MenB vaccine. Exclusion criteria were previous meningococcal disease (or household or intimate contact with anyone with meningococcal disease), allergy to vaccine components, severe acute or chronic disease, immune dysfunction, receipt of blood products, planned receipt of nonstudy vaccines within 30 days of the study vaccines, enrolment in another clinical trial, recent antibiotic use, being a family member of research staff or antipyretic use within 6 hours before enrolment.

Written informed consent was obtained from participant's parents or legal guardians. Ethics approval was obtained from Oxfordshire Research Ethics Committee B (reference no. 09/H0605/89). The study was conducted from January to December 2010.

\section{Procedures}

Participants who had previously received 4 doses of $4 \mathrm{CMenB}$ or rMenB in the original study had blood samples taken before and 30 days after a booster dose of the respective vaccines (Figure 1). Participants previously given a single dose of $4 \mathrm{CMenB}$ or rMenB in the original study had 2 doses of the respective vaccine administered about 60 days apart. Control participants received $4 \mathrm{CMenB}$ at 40 and 42 months of age, thereby receiving 2 doses of $4 \mathrm{CMenB}$ at least 2 months apart, the approved dosing schedule for this age group. All participants had blood samples taken at enrolment and 30 days after each vaccination.

Both vaccines were manufactured and supplied by Novartis Vaccines and Diagnostics. Both contained $50 \mu \mathrm{g}$ each of NadA allele 3, NHBA-GNA (genome-derived Neisseria antigen) 1030 fusion protein (containing NHBA peptide variant 2) and GNA2091-fHbp fusion protein (containing fHbp variant 1.1) as well as $1.5 \mathrm{mg}$ aluminum hydroxide and $10 \mathrm{mM}$ histidine. The $4 \mathrm{CMenB}$ vaccine also contained $25 \mu \mathrm{g}$ of detoxified outer membrane vesicles from Neisseria meningitidis strain NZ98/254 (expressing the immunodominant antigen PorA [porin A] serosubtype P1.4). The vaccines were $0.5 \mathrm{~mL}$ in volume and were administered by intramuscular injection into the deltoid area of the nondominant arm. Participants also received a combination diphtheria-toxoid, tetanus-toxoid,

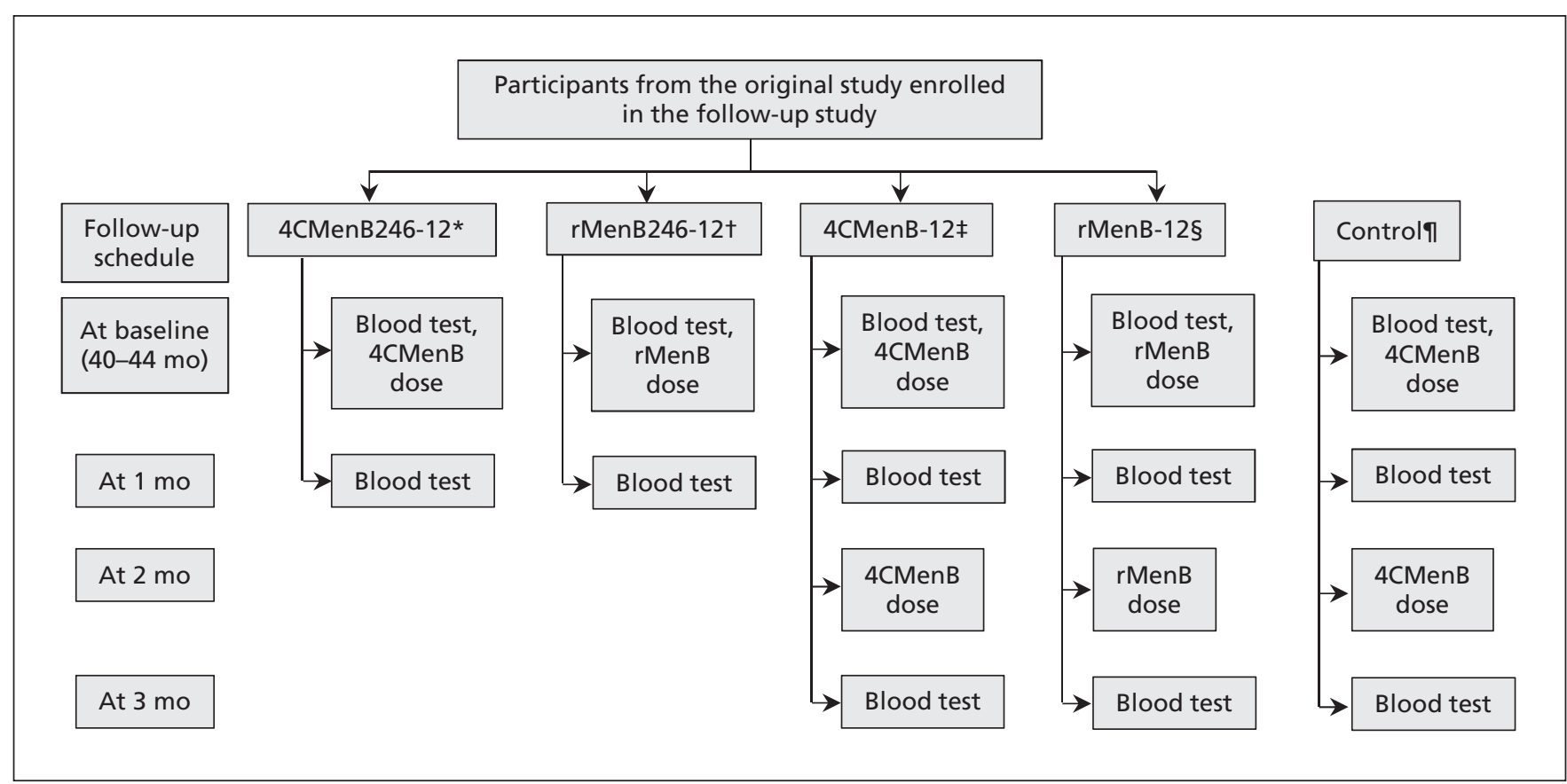

Figure 1: Study design. *Participants who received 4 doses of multicomponent serogroup B meningococcal (4CMenB) vaccine at 2, 4, 6 and 12 months. tParticipants who received 4 doses of recombinant protein serogroup B meningococcal (rMenB) vaccine at 2, 4, 6 and 12 months. $¥$ Participants who received 1 dose of 4 CMenB vaccine at 12 months. §Participants who received 1 dose of rMenB vaccine at 12 months. ПParticipants with no previous exposure to $4 C M e n B$ or rMenB vaccines. 
acellular pertussis and inactivated polio vaccine (Repevax, Sanofi Pasteur MSD) and a combination measles, mumps and rubella vaccine (MMRvaxPro, Sanofi Pasteur MSD) at the final study visit. These vaccines were administered to keep participants vaccinated according to the routine schedule in the United Kingdom and did not form part of the study evaluation.

\section{Immunogenicity evaluation}

Sera were analyzed at the Vaccine Evaluation Unit of the Health Protection Agency in Manchester, UK, for serum bactericidal activity using a human complement source as previously described. ${ }^{9}$ Laboratory staff were unaware of participants' group allocation. The correlate of protection was a human complement serum bactericidal activity (hSBA) titre of 1:4 or greater. As previously described, ${ }^{3}$ reference hSBA strains were used to assess the immunogenicity of specific vaccine components: fHbp was assessed by strain 44/76-SL, NadA by strain 5/99 and PorA (the immunodominant antigen in the outer membrane vesicle) by NZ98/254 (Table 1). We used a novel strain (M10713) to assess the immunogenicity of NHBA because this expresses NHBA cross-reactive to that contained in the vaccine (peptide 10) but is mismatched for the vaccine components fHbp (subvariant 2.24) and PorA (16-3) and does not contain the gene for NadA. Therefore, in accordance with the principle underlying the use of the existing reference strains, any increase in hSBA titres against M10713 following vaccination may be attributed to the NHBA component of the vaccine. We assessed immunogenicity against additional strains (M00-242922, M01-240101, M01240364) to evaluate the impact of antigenic variation and expression on susceptibility to vaccineinduced antibodies (Table 1). We specifically selected strain M01-240355 as a strain likely to be relatively resistant to vaccine-induced bactericidal antibodies because it was mismatched for all vaccine antigens.

\section{Reactogenicity evaluation}

Each day in the week after vaccination, parents recorded their child's axillary temperature, local reactions at the site of vaccination (pain, erythema, swelling and induration), solicited systemic reactions (fever, irritability, vomiting, diarrhea, sleepiness, anorexia, arthralgia, headache and rash) and the use of analgesic and antipyretic medication. Reactions were classified as severe if tenderness prevented daily activity, local erythema and induration were greater than $50 \mathrm{~mm}$ in diameter or the child's fever after vaccination was $40^{\circ} \mathrm{C}$ or higher.

\section{Outcome measures}

The primary outcome measure for immunogenicity was the percentage of children with an hSBA titre of 1:4 or greater among those who received 4 doses of either vaccine. The primary safety objective was to assess the safety and tolerability of 1 or 2 booster doses of these vaccines.

Secondary objectives were assessment of the persistence of bactericidal antibodies following vaccination with 1 dose of $4 \mathrm{CMenB}$ or rMenB at 12 months of age, increases in hSBA titres following 1 or 2 booster doses and adverse reactions following a 2-dose "catch-up" vaccination schedule of 4CMenB administered at enrolment.

\section{Statistical analysis}

We calculated the percentages of children in each study group with hSBA titres of 1:4 or greater, along with 2-sided 95\% ClopperPearson confidence intervals (CIs). ${ }^{11}$ Similarly, we calculated geometric mean titres of bactericidal antibodies and determined $95 \%$ CIs by exponentiating (base 10) the corresponding means and 95\% CI of the log $10 \mathrm{hSBA}$ titre. Geometric mean ratios of hSBA geometric mean titres after vaccination to baseline and $95 \%$ CIs were also calculated. For these calculations, we allocated a value of 1 to hSBA titres below the lower limit of detection of 2 .

The primary population for immunogenicity analysis was the intention-to-treat group, consisting of all participants who were randomly assigned, received a dose of either vaccine and provided at least 1 evaluable serum sample. Safety analysis was conducted on a population consisting of all participants who received a dose of either vaccine and provided safety data after baseline.

The sample size of the follow-up participants was determined by the number of participants who completed the original study at the Oxford site. All comparisons were descriptive. However, we calculated that if the percentage of participants with hSBA titres of $1: 4$ or greater was $40 \%$ in a follow-up group and 5\% in the control group, a sample size of 30 participants in the follow-up group and 50 participants in the control group would be required for $95 \%$ power to show superiority.

\section{Results}

Of the 125 participants who completed the original study at the Oxford site, 70 were enrolled in the follow-up study, of whom 68 were included in the intention-to-treat analysis. In addition, 43 MenB vaccine-naive participants were enrolled, of whom 40 were included in the intention-to- 


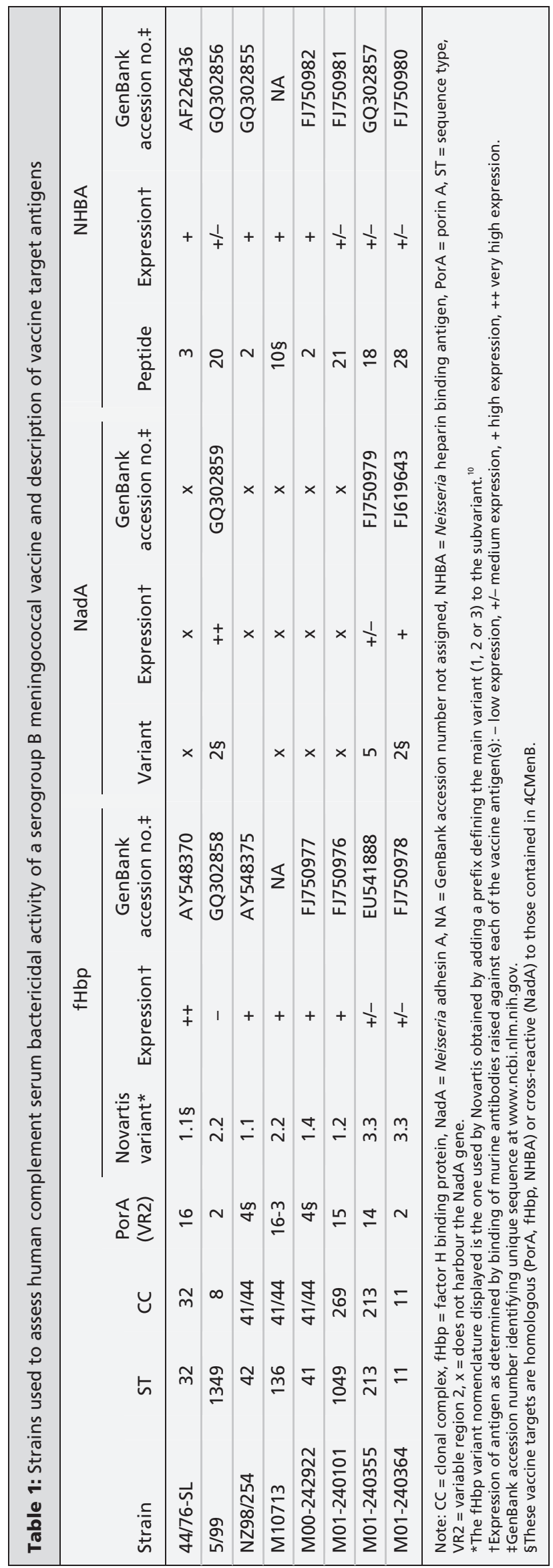


treat analysis (Table 2). The demographic characteristics of the participants were similar across all groups. The median age at enrolment was 41 (range 40-44) months, 50\% were male (compared with $57 \%$ in the original study) and $92 \%$ of participants were white (compared with $94 \%$ in the original study).

\section{Immunogenicity}

Waning of antibodies was observed at baseline following vaccination in early infancy with either vaccine. At least $65 \%$ of participants who received $4 \mathrm{CMenB}$ at 2, 4, 6 and 12 months had hSBA titres of 1:4 or greater at baseline for strains 44/76-SL (evaluating fHbp), 5/99 (NadA) and M10713 (NHBA) compared with $41 \%$ for NZ98/254 (PorA) (Table 3; Appendix 1, available at www.cmaj.ca/lookup/suppl/doi:10.1503 /cmaj.130257/-/DC1). Among participants who received rMenB at 2, 4, 6 and 12 months of age, at least $43 \%$ showed hSBA titres of $1: 4$ or greater for these strains, except for NZ98/254 (PorA) (3\%). As expected, the percentage of participants with hSBA titres of 1:4 or greater for strain M01-240355 was low, regardless of the vaccine received.

At baseline, $25 \%$ and $38 \%$ of children who received $4 \mathrm{CMenB}$ at 12 months had hSBA titres of 1:4 or greater for strains M10713 (NHBA) and 44/76-SL (fHbp), respectively, whereas for strains 5/99 (NadA) and NZ98/254 (PorA), these proportions were $0 \%$ (Table 3, Appendix 1).

Administration of a booster dose of $4 \mathrm{CMenB}$ at enrolment resulted in $86 \%-100 \%$ of 4CMenB-primed participants achieving hSBA titres of 1:4 or greater for all strains except M01-240355.

In MenB vaccine-naive children, administration of 2 doses of 4CMenB 2 months apart resulted in $89 \%-100 \%$ achieving hSBA titres of 1:4 or greater for all strains except M01-240355 $(69 \%)$. More than half of these children had hSBA titres greater than 1:4 to strains 44/76-SL (fHbp), M10713 (NHBA), M01-240101 and M01-240364 at baseline, with hSBA geometric mean titres similar to those of participants who received $4 \mathrm{CMenB}$ at 2, 4, 6 and 12 months. The geometric rise in hSBA titres for all these strains following a 4CMenB booster dose was higher in the group that received $4 \mathrm{CMenB}$ at 2, 4, 6 and 12 months than in controls, as determined by nonoverlapping CIs (Table 4). The hSBA geometric mean titres for all time points in this and the original study are displayed in Appendix 2 (available at www.cmaj.ca/lookup/suppl/doi:10 .1503/cmaj.130257/-/DC1).

\section{Reactogenicity}

Most recipients of either vaccine had local pain and erythema at the injection site, but few reactions were severe (Appendices 3 and 4, available at www.cmaj.ca/lookup/suppl/doi:10.1503/cmaj .130257/-/DC1). Few participants reported fever.

One serious adverse event was experienced by a participant in the control group in whom otitis media developed 12 days after vaccination with $4 \mathrm{CMenB}$, followed by cervical lymphadenitis. He required hospital admission for intravenous antibiotics. This was considered unrelated to the vaccination.

Table 2: Number of participants included in the original study and the follow-up study

\begin{tabular}{|c|c|c|c|c|c|c|c|c|}
\hline \multirow[b]{3}{*}{ Group } & \multicolumn{2}{|c|}{ Original study } & \multicolumn{6}{|c|}{ Follow-up study } \\
\hline & & & \multicolumn{3}{|c|}{ At baseline } & \multirow{2}{*}{$\begin{array}{c}\text { At } 1 \text { mo } \\
\text { Included in ITT } \\
\text { analysis for } \\
\text { immunogenicity } t\end{array}$} & \multirow{2}{*}{$\begin{array}{l}\text { At } 2 \text { mo } \\
\begin{array}{l}\text { Received } \\
\text { second } \\
\text { dose }\end{array}\end{array}$} & \multirow{2}{*}{$\begin{array}{c}\text { At } 3 \text { mo } \\
\text { Included in ITT } \\
\text { analysis for } \\
\text { immunogenicity } †\end{array}$} \\
\hline & $\begin{array}{l}\text { Enrolled } \\
\text { (Oxford)* }\end{array}$ & $\begin{array}{c}\text { Completed } \\
\text { (Oxford)* }\end{array}$ & Enrolled & $\begin{array}{c}\text { Included in ITT } \\
\text { analysis for } \\
\text { persistencet }\end{array}$ & $\begin{array}{l}\text { Received } \\
\text { first dose }\end{array}$ & & & \\
\hline 4CMenB246-12‡ & $50(46)$ & $45(41)$ & 19 & 17 & 19 & 19 & NA & NA \\
\hline rMenB246-12§ & $48(44)$ & $44(40)$ & 29 & 29 & 29 & 28 & NA & NA \\
\hline 4CMenB-12ף & $24(22)$ & $23(21)$ & 8 & 8 & 8 & 7 & 8 & 8 \\
\hline rMenB-12** & $25(23)$ & $24(23)$ & 14 & 14 & 14 & 13 & 14 & 13 \\
\hline Controlt† & NA & NA & 43 & 40 & 42 & 38 & 41 & 36 \\
\hline \multicolumn{9}{|c|}{ 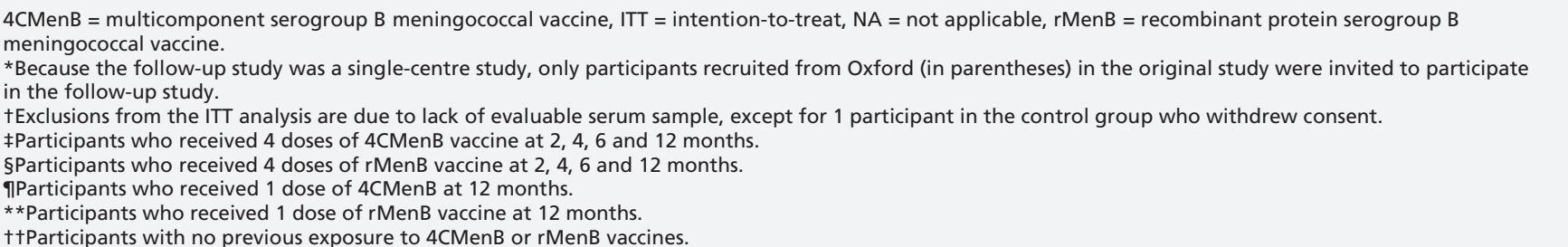 } \\
\hline
\end{tabular}


Table 3: Participants with human complement serum bactericidal activity titres of 1:4 or greater, intention-to-treat population

\begin{tabular}{|c|c|c|c|c|c|c|c|}
\hline \multirow{2}{*}{$\begin{array}{l}\text { Strain } \\
\text { (antigen } \\
\text { assessed) }\end{array}$} & \multirow[b]{2}{*}{ Group } & \multicolumn{2}{|c|}{ At baseline } & \multicolumn{2}{|c|}{ At $1 \mathrm{mo}$} & \multicolumn{2}{|c|}{ At $3 \mathrm{mo}$} \\
\hline & & $\%(95 \% \mathrm{Cl})$ & $n / N$ & $\%(95 \% \mathrm{Cl})$ & $n / N$ & $\%(95 \% \mathrm{Cl})$ & $n / N$ \\
\hline \multirow{5}{*}{$\begin{array}{l}\text { 44/76-SL } \\
\text { (fHbp) }\end{array}$} & 4CMenB246-12* & $65(38-86)$ & $11 / 17$ & $100(82-100)$ & $19 / 19$ & NA & NA \\
\hline & rMenB246-12† & $45(26-64)$ & $13 / 29$ & $100(88-100)$ & $28 / 28$ & NA & NA \\
\hline & 4CMenB-12‡ & $38 \quad(9-76)$ & $3 / 8$ & $100(59-100)$ & $7 / 7$ & $100(63-100)$ & $8 / 8$ \\
\hline & rMenB-12§ & $57(29-82)$ & $8 / 14$ & $100(75-100)$ & $13 / 13$ & $100(75-100)$ & $13 / 13$ \\
\hline & Controlף & $63(46-77)$ & $25 / 40$ & 89 (75-97) & $34 / 38$ & $100(90-100)$ & $36 / 36$ \\
\hline \multirow{5}{*}{$\begin{array}{l}5 / 99 \\
(\mathrm{NadA})\end{array}$} & 4CMenB246-12* & 76 (50-93) & $13 / 17$ & $100(81-100)$ & $18 / 18$ & NA & NA \\
\hline & rMenB246-12† & $43(24-63)$ & $12 / 28$ & $100(88-100)$ & $28 / 28$ & NA & NA \\
\hline & 4CMenB-12 $\ddagger$ & $0 \quad(0-37)$ & $0 / 8$ & $100(59-100)$ & $7 / 7$ & $100(63-100)$ & $8 / 8$ \\
\hline & rMenB-12§ & $57(29-82)$ & $8 / 14$ & $100(75-100)$ & $13 / 13$ & $100(75-100)$ & $13 / 13$ \\
\hline & Controlף & $3 \quad(0-13)$ & $1 / 40$ & $76(60-89)$ & $29 / 38$ & $100(90-100)$ & $36 / 36$ \\
\hline \multirow{5}{*}{$\begin{array}{l}\text { NZ98/254 } \\
\text { (PorA) }\end{array}$} & 4CMenB246-12* & $41(18-67)$ & 7/17 & 89 (67-99) & $17 / 19$ & NA & NA \\
\hline & rMenB246-12† & $3 \quad(0-18)$ & $1 / 29$ & $14 \quad(4-33)$ & $4 / 28$ & NA & NA \\
\hline & 4CMenB-12 & $0 \quad(0-37)$ & $0 / 8$ & $100(59-100)$ & $7 / 7$ & $100(63-100)$ & $8 / 8$ \\
\hline & rMenB-12§ & $7 \quad(0-34)$ & $1 / 14$ & $15 \quad(2-45)$ & $2 / 13$ & $15 \quad(2-45)$ & $2 / 13$ \\
\hline & Controlף & $0 \quad(0-9)$ & $1 / 40$ & $66(49-80)$ & $25 / 38$ & 94 (81-99) & $34 / 36$ \\
\hline \multirow{5}{*}{$\begin{array}{l}\text { M10713 } \\
\text { (NHBA) }\end{array}$} & 4CMenB246-12* & $67(38-88)$ & $10 / 15$ & $94(73-100)$ & $17 / 18$ & NA & NA \\
\hline & rMenB246-12† & $68(48-84)$ & $19 / 28$ & $96(82-100)$ & $27 / 28$ & NA & NA \\
\hline & 4CMenB-12 & $25 \quad(3-65)$ & $2 / 8$ & $86(42-100)$ & $6 / 7$ & $100(63-100)$ & $8 / 8$ \\
\hline & rMenB-12§ & $54(25-81)$ & $7 / 13$ & $100(75-100)$ & $13 / 13$ & $83(52-98)$ & $10 / 12$ \\
\hline & Controlף & $68(51-81)$ & $27 / 40$ & $76(60-89)$ & $29 / 38$ & 89 (74-97) & $32 / 36$ \\
\hline \multirow{5}{*}{$\begin{array}{l}\text { M00- } \\
242922\end{array}$} & 4CMenB246-12* & $53(28-77)$ & 9/17 & $100(81-100)$ & $18 / 18$ & NA & NA \\
\hline & rMenB246-12† & $18 \quad(6-37)$ & $5 / 28$ & 75 (55-89) & $21 / 28$ & NA & NA \\
\hline & 4CMenB-12 & $0 \quad(0-37)$ & $0 / 8$ & $100(59-100)$ & $7 / 7$ & $100(63-100)$ & $8 / 8$ \\
\hline & rMenB-12§ & $8 \quad(0-36)$ & $1 / 13$ & $54(25-81)$ & $7 / 13$ & $42(15-72)$ & $5 / 12$ \\
\hline & Controlף & $18 \quad(8-34)$ & $7 / 39$ & $49(32-66)$ & $18 / 37$ & 94 (81-99) & $33 / 35$ \\
\hline \multirow{5}{*}{$\begin{array}{l}\text { M01- } \\
240101\end{array}$} & 4CMenB246-12* & $71(44-90)$ & $12 / 17$ & $100(81-100)$ & $18 / 18$ & NA & NA \\
\hline & rMenB246-12† & $71(51-87)$ & $20 / 28$ & $100(88-100)$ & $28 / 28$ & NA & NA \\
\hline & 4CMenB-12 $\ddagger$ & $63(24-91)$ & $5 / 8$ & $100(59-100)$ & $7 / 7$ & $100(63-100)$ & $8 / 8$ \\
\hline & rMenB-12§ & 77 (46-95) & $10 / 13$ & $100(75-100)$ & $13 / 13$ & $100(74-100)$ & $12 / 12$ \\
\hline & Controlף & $74(58-87)$ & $29 / 39$ & $97(86-100)$ & $36 / 37$ & $100(90-100)$ & $35 / 35$ \\
\hline \multirow{5}{*}{$\begin{array}{l}\text { M01- } \\
240355\end{array}$} & 4CMenB246-12* & $6 \quad(0-29)$ & $1 / 17$ & $50(26-74)$ & $9 / 18$ & NA & NA \\
\hline & rMenB246-12† & $25(11-45)$ & $7 / 28$ & $29(13-49)$ & $8 / 28$ & NA & NA \\
\hline & 4CMenB-12 & $25 \quad(3-65)$ & $2 / 8$ & $71(29-96)$ & $5 / 7$ & $63(24-91)$ & $5 / 8$ \\
\hline & rMenB-12§ & $15 \quad(2-45)$ & $2 / 13$ & $31 \quad(9-61)$ & $4 / 13$ & $8 \quad(0-38)$ & $1 / 12$ \\
\hline & Controlף & $18 \quad(8-34)$ & $7 / 39$ & $30(16-47)$ & $11 / 37$ & $69(51-83)$ & $24 / 35$ \\
\hline \multirow{5}{*}{$\begin{array}{l}\text { M01- } \\
240364\end{array}$} & 4CMenB246-12* & 88 (64-99) & $15 / 17$ & $94(73-100)$ & $17 / 18$ & NA & NA \\
\hline & rMenB246-12† & $61(41-78)$ & $17 / 28$ & $82(63-94)$ & $23 / 28$ & NA & NA \\
\hline & 4CMenB-12 $\ddagger$ & $50(16-84)$ & $4 / 8$ & $100(59-100)$ & $7 / 7$ & $100(63-100)$ & $8 / 8$ \\
\hline & rMenB-12§ & 77 (46-95) & $10 / 13$ & $100(75-100)$ & $13 / 13$ & $100(74-100)$ & $12 / 12$ \\
\hline & Controlף & $82(66-92)$ & $31 / 38$ & 86 (71-95) & $32 / 37$ & $100(90-100)$ & $34 / 34$ \\
\hline
\end{tabular}

$\mathrm{Cl}=$ confidence interval, $\mathrm{fHbp}=$ factor $\mathrm{H}$ binding protein, $\mathrm{NA}=$ not applicable, $\mathrm{NadA}=$ Neisseria adhesin $\mathrm{A}, \mathrm{NHBA}=\mathrm{Neisseria}$ heparin binding antigen,

PorA = porin A.

*Participants who received 4 doses of $4 C M e n B$ vaccine at $2,4,6$ and 12 months.

†Participants who received 4 doses of $r M e n B$ vaccine at 2, 4, 6 and 12 months.

¥Participants who received 1 dose of 4 CMenB at 12 months.

§Participants who received 1 dose of rMenB vaccine at 12 months.

ПParticipants with no previous exposure to 4 CMenB or $r$ MenB vaccines. 
Table 4: Geometric mean titres and geometric mean ratios of human complement serum bactericidal activity, intention-to-treat population

\begin{tabular}{|c|c|c|c|c|c|c|}
\hline \multirow{2}{*}{$\begin{array}{l}\text { Strain (antigen } \\
\text { assessed) }\end{array}$} & \multirow[b]{2}{*}{ Group } & \multicolumn{3}{|c|}{ Geometric mean titres $(95 \% \mathrm{Cl})$} & \multicolumn{2}{|c|}{ Geometric mean ratios $(95 \% \mathrm{Cl})$} \\
\hline & & At baseline & At $1 \mathrm{mo}$ & At $3 \mathrm{mo}$ & 1 mo:baseline & 3 mo:baseline \\
\hline \multirow[t]{5}{*}{ 44/76-SL (fHbp) } & 4CMenB 246-12* & $5.3(3.3-8.8)$ & $89(68-116)$ & NA & $17(11-27)$ & NA \\
\hline & rMenB246-12† & $3.2(2.4-4.5)$ & $99(62-158)$ & NA & $30(18-50)$ & NA \\
\hline & 4CMenB-12‡ & $3.5(0.9-14)$ & $76(27-210)$ & $145(74-283)$ & $21(4.2-110)$ & $42(12-147)$ \\
\hline & rMenB-12§ & $3.6(2.1-6.2)$ & $94(46-192)$ & $127(81-200)$ & $27(13-57)$ & $37(21-64)$ \\
\hline & Controlף & $4.2(3.2-5.6)$ & $12(8.0-19)$ & $88(66-117)$ & $3.2(2.1-5.0)$ & $20(15-28)$ \\
\hline \multirow[t]{5}{*}{$5 / 99$ (NadA) } & 4CMenB246-12* & $28(9.4-83)$ & $1708(774-3771)$ & NA & $70(29-167)$ & NA \\
\hline & rMenB246-12† & $5.1(2.3-11)$ & $778(461-1311)$ & NA & $148(90-245)$ & NA \\
\hline & 4CMenB-12f & $1.0(1.0-1.0)$ & $509(178-1456)$ & $2413(988-5892)$ & 509 (178-1456) & $2413(988-5892)$ \\
\hline & rMenB-12§ & $9.6(4.2-22)$ & 2379 (995-5685) & $5240(3216-8538)$ & 299 (129-694) & 501 (143-1748) \\
\hline & Controlף & $1.1(0.7-1.8)$ & $22(13-36)$ & 1019 (760-1367) & $19(10-36)$ & 910 (594-1394) \\
\hline \multirow[t]{5}{*}{ NZ98/254 (PorA) } & 4CMenB246-12* & $2.8(1.4-5.6)$ & $47(20-107)$ & NA & $20(7.7-51)$ & NA \\
\hline & rMenB246-12† & $1.1(1.0-1.2)$ & $1.6(1.0-2.6)$ & NA & $1.5(1.0-2.4)$ & NA \\
\hline & 4CMenB-12‡ & $1.0(1.0-1.0)$ & $148(66-330)$ & 65 (22-189) & $148(66-330)$ & 65 (22-189) \\
\hline & rMenB-12§ & $1.2(0.9-1.7)$ & $1.7(0.8-3.8)$ & $1.9(0.9-3.7)$ & $1.4(0.6-3.1)$ & $1.5(0.7-3.1)$ \\
\hline & Controlף & $1.0(1.0-1.0)$ & $7.7(4.6-13)$ & $47(31-72)$ & $7.6(4.5-13)$ & $47(31-74)$ \\
\hline \multirow[t]{5}{*}{ M10713 (NHBA) } & 4CMenB246-12* & $5.3(2.3-12)$ & $39(22-69)$ & NA & $10(4.3-24)$ & NA \\
\hline & rMenB246-12† & $9.2(4.9-17)$ & $38(24-59)$ & NA & $4.7(2.3-9.4)$ & NA \\
\hline & 4CMenB-12 & $3.0(0.8-12)$ & $30(9.5-96)$ & $36(21-61)$ & $10(2.0-49)$ & $12(3.0-48)$ \\
\hline & rMenB-12§ & $3.3(1.6-6.6)$ & 35 (19-67) & $21(7.4-60)$ & $11(4.2-30)$ & $5.5(1.5-20)$ \\
\hline & Controlף & $8.8(5.2-15)$ & $11(6.7-19)$ & $33(22-51)$ & $1.3(0.9-1.8)$ & $3.9(2.1-7.2)$ \\
\hline \multirow[t]{5}{*}{ M00-242922 } & 4CMenB246-12* & $3.5(1.7-7.0)$ & $64(27-155)$ & NA & $22(8.3-56)$ & NA \\
\hline & rMenB246-12† & $1.6(1.2-2.2)$ & $7.0(4.4-11)$ & NA & $4.6(2.9-7.5)$ & NA \\
\hline & 4CMenB-12‡ & $1.0(1.0-1.0)$ & 206 (33-1308) & $53(21-131)$ & $206(33-1308)$ & $53(21-131)$ \\
\hline & rMenB-12§ & $1.2(0.9-1.7)$ & $4.6(1.6-13)$ & $3.0(1.6-5.7)$ & $3.8(1.5-9.6)$ & $2.4(1.2-5.0)$ \\
\hline & Controlף & $1.4(1.2-1.8)$ & $3.6(2.6-5.2)$ & $31(22-44)$ & $2.7(2.0-3.8)$ & $23(16-34)$ \\
\hline \multirow[t]{5}{*}{ M01-240101 } & 4CMenB246-12* & $7.6(4.3-13)$ & $159(80-314)$ & NA & $20(6.8-59)$ & NA \\
\hline & rMenB246-12† & $7.0(4.0-12)$ & $76(40-143)$ & NA & $11(5.7-23)$ & NA \\
\hline & 4CMenB-12 & $4.0(1.7-9.2)$ & $59(14-248)$ & $92(40-213)$ & $16(2.4-105)$ & $23(5.4-100)$ \\
\hline & rMenB-12§ & $12(3.4-41)$ & $82(35-190)$ & $43(21-89)$ & $6.9(2.8-17)$ & $4.2(1.1-16)$ \\
\hline & Controlף & $6.6(4.4-9.9)$ & $87(46-165)$ & $207(120-358)$ & $12(6.5-23)$ & $33(17-64)$ \\
\hline \multirow[t]{5}{*}{ M01-240355 } & 4CMenB246-12* & $1.2(0.8-1.7)$ & $5.0(2.6-9.3)$ & NA & $4.2(2.2-8.2)$ & NA \\
\hline & rMenB246-12† & $1.8(1.2-2.8)$ & $1.9(1.3-2.8)$ & NA & $1.0(0.7-1.6)$ & NA \\
\hline & 4CMenB-12‡ & $1.7(0.9-3.0)$ & $5.9(3.1-11)$ & $5.5(1.8-17)$ & $3.3(1.3-8.5)$ & $3.3(1.4-7.7)$ \\
\hline & rMenB-12§ & $1.4(0.8-2.4)$ & $1.7(1.0-2.9)$ & $1.3(0.8-2.0)$ & $1.2(0.8-1.7)$ & $0.9(0.6-1.3)$ \\
\hline & Controlף & $1.5(1.2-2.0)$ & $2.5(1.5-4.1)$ & $6.2(3.6-11)$ & $1.7(1.2-2.5)$ & $4.1(2.7-6.3)$ \\
\hline \multirow[t]{5}{*}{ M01-240364 } & 4CMenB246-12* & $17(8.3-36)$ & $134(56-322)$ & NA & $8.3(3.0-23)$ & NA \\
\hline & rMenB246-12† & $6.5(3.6-12)$ & $41(17-96)$ & NA & $6.2(2.6-15)$ & NA \\
\hline & 4CMenB-12‡ & $5.0(1.6-16)$ & $52(14-193)$ & $107(26-443)$ & $8.2(1.6-43)$ & $21(5.0-91)$ \\
\hline & rMenB-12§ & $12(4.9-31)$ & 275 (144-528) & 616 (191-1980) & $22(11-44)$ & 56 (18-172) \\
\hline & Controlף & $9.4(6.5-14)$ & $11(7.6-16)$ & $104(68-159)$ & $1.2(0.8-1.7)$ & $11(6.4-18)$ \\
\hline \multicolumn{7}{|c|}{ 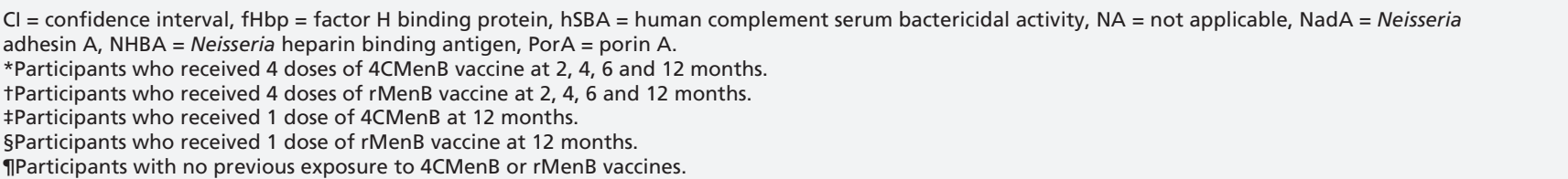 } \\
\hline
\end{tabular}




\section{Interpretation}

The 4CMenB vaccine was licensed for use in Europe in January 2013, with approved dosage schedules including the 2, 4, 6, 12 month and 40, 42 month schedules evaluated in this study. In contrast to the single dose of $4 \mathrm{CMenB}$ given at 12 months of age to one of our study groups, the licensed indication for children 1-2 years of age is 2 doses of $4 \mathrm{CMenB}$ at least 2 months apart, with a booster dose given 1-2 years later.

Among children who received $4 \mathrm{CMenB}$ at 2, 4,6 , and 12 months of age in the original study, by 40 months of age the proportions with hSBA titres of 1:4 or greater were $65 \%$ for strain 44/76SL (fHbp), 76\% for strain 5/99 (NadA) and 41\% for strain NZ98/254. These proportions represent a decline from 13 months of age $(100 \%, 93 \%$ and $96 \%$, respectively). ${ }^{3}$ However, a good response to a booster dose of vaccine was observed. Experience with other meningococcal vaccines has shown that waning of bactericidal antibody titres was associated with a decline in vaccine effectiveness following infant vaccination with serogroup $\mathrm{C}$ meningococcal conjugate vaccines, ${ }^{12}$ adolescent vaccination with an investigational outer membrane vesicle vaccine in Norway $^{13}$ and infant vaccination with the New Zealand outer membrane vesicle vaccine. ${ }^{10,14}$ Predicting the potential impact of a decline in bactericidal antibodies on vaccine effectiveness following vaccination with $4 \mathrm{CMenB}$ is less straightforward. The serogroup $\mathrm{C}$ meningococcal conjugate and outer membrane vesicle vaccines generate an immune response primarily directed against a single antigen (the capsular polysaccharide and PorA, respectively), whereas $4 \mathrm{CMenB}$ is a multicomponent vaccine that aims to induce antibodies against each component. The immunogenicity of $4 \mathrm{CMenB}$ antigens can therefore be assessed only by use of multiple meningococcal strains, and there is considerable interstrain variation in the rate of decline of hSBA titres. It is unclear whether this reflects true differences in the persistence of efficacy for each vaccine component, or if this simply reflects different susceptibilities of the strains to killing in the hSBA assay. However, it does raise the possibility that, after vaccination, susceptibility to meningococcal infection could develop at different rates for different strains, depending on whether they are expressing proteins recognized by persistent or waning antibodies. It is also notable that the bactericidal antibody concentrations against strains 44/76-SL (fHbp) and M10713 (NHBA) were similar in children previously vaccinated with $4 \mathrm{CMenB}$ and those who were MenB vaccine-naive. Although this may suggest the acquisition of natural immunity against these strains (evident in more than twothirds of the naive cohort), these children had a relatively poor increase in bactericidal antibodies following a single dose of $4 \mathrm{CMenB}$ when compared with the vaccine-primed children. This suggests previous vaccination with $4 \mathrm{CMenB}$ has resulted in effective priming of the immune systems of vaccine recipients, but whether this means they have any greater protection against serogroup B meningococcal disease than MenB vaccine-naive children with the same hSBA titres remains to be seen.

A related issue is the proportion of serogroup B meningococcal strains likely to be prevented by vaccination with $4 \mathrm{CMenB}$. Because the use of the hSBA assay for a very large panel of strains is not practical for technical reasons, attempts to predict coverage of meningococcal vaccines in development using surrogate assays more suitable for high throughput and standardization have been used. One example of this is the Meningococcal Antigen Typing System developed by Novartis. ${ }^{15}$ The system evaluates a representative panel of meningococcal strains to correlate the strength of binding (i.e., relative potency) of vaccine antigen-specific immunoglobulin $\mathrm{G}$ to the presence or absence of strainspecific bactericidal activity on the hSBA assay (performed using pooled serum obtained after vaccination at age $13 \mathrm{mo}$ ). Regional reference laboratories can then calculate the percentage of local invasive strains where the relative potency is above this positive bactericidal threshold for 1 or more of fHbp, NadA or NHBA (or where the PorA variant is homologous to the outer membrane vesicle component of $4 \mathrm{CMenB}$ ). Using this technique, the Meningococcal Antigen Typing System predicts vaccination coverage of $78 \%$ of invasive serogroup B meningococcal strains in Europe, ${ }^{16} 76 \%$ in Australia ${ }^{17}$ and $66 \%$ in Canada..$^{18}$ Provisional data from the African meningitis belt predict $100 \%$ coverage for 9 serogroup $\mathrm{X}$ meningococcal strains. ${ }^{19}$ The demonstration of waning bactericidal antibodies in this study suggests that pooled sera obtained 2-3 years after vaccination would kill a smaller proportion of strains in the assay than that obtained at 13 months ( 1 mo after vaccination).

The above issues address the generation of direct protection by vaccination with $4 \mathrm{CMenB}$. However, the impact of this vaccine will be determined at least as much by the presence or absence of herd immunity, which has been especially important for other meningococcal vaccines to ensure sustained protection in the face of waning immunity. Studies are currently underway to determine whether $4 \mathrm{CMenB}$ is likely to 
influence nasopharyngeal carriage and hence induce herd immunity by reducing circulation of the organism (NCT01214850). If not, the maintenance of adequate levels of bactericidal antibodies to provide direct protection may be even more critical than for conjugate vaccines. If vaccination with $4 \mathrm{CMenB}$ does influence nasopharyngeal carriage, then it will be important to monitor the population of "carried" strains to assess for the potential emergence of strains either lacking the vaccine antigens or expressing these at low levels, as this could herald the emergence of meningococci less susceptible to prevention by $4 \mathrm{CMenB}$.

\section{Limitations}

There were a number of limitations in this study, including the relatively small numbers of participants and the fact that $45 \%$ of participants from the original study who were eligible to take part in the follow-up study did not do so. There is a potential for bias, in that children who tolerated the vaccine better in the original study may have been more likely to participate in the follow-up study. Furthermore, the control group underrecruited. Follow-up studies are currently being conducted from the larger phase IIb and III studies (NCT00944034, NCT00847145) and will provide additional data on antibody persistence and tolerability. There is as yet no information on persistence after booster doses in midchildhood; these data will become available soon because children from this study will be revisited when they reach 5 years of age (NCT01027351).

\section{Conclusion}

Consistent with other vaccines against meningococcal disease, a waning of hSBA titres was observed after infant vaccination with $4 \mathrm{CMenB}$. A booster dose during preschool years was well tolerated. If $4 \mathrm{CMenB}$ were to be introduced into a routine vaccination schedule, measures such as adequate disease surveillance would be important to determine whether waning of antibodies might influence the effectiveness of a vaccination campaign against this bacterium.

\section{References}

1. Summary of opinion: Bexsero. London (UK): European Medicines Agency; 2012. Available: www.ema.europa.eu/docs/en_GB /document_library/Summary_of_opinion_-_Initial_authorisation /human/002333/WC500134836.pdf (accessed 2013 May 8).

2. Gossger N, Snape MD, Yu LM, et al. Immunogenicity and tolerability of recombinant serogroup B meningococcal vaccine administered with or without routine infant vaccinations according to different immunization schedules: a randomized controlled trial. JAMA 2012;307:573-82.

3. Findlow J, Borrow R, Snape MD, et al. Multicenter, open-label, randomized phase II controlled trial of an investigational recombinant meningococcal serogroup B vaccine with and without outer membrane vesicles, administered in infancy. Clin Infect Dis 2010;51:1127-37.
4. Snape MD, Dawson T, Oster P, et al. Immunogenicity of two investigational serogroup $\mathrm{B}$ meningococcal vaccines in the first year of life: a randomized comparative trial. Pediatr Infect Dis $J$ 2010;29:e71-9.

5. Santolaya ME, O'Ryan ML, Valenzuela MT, et al. Immunogenicity and tolerability of a multicomponent meningococcal serogroup B (4CMenB) vaccine in healthy adolescents in Chile: a phase $2 \mathrm{~b} / 3$ randomised, observer-blind, placebo-controlled study. Lancet 2012;379:617-24.

6. Vesikari T, Esposito S, Prymula R, et al. Immunogenicity and safety of an investigational multicomponent, recombinant, meningococcal serogroup B vaccine (4CMenB) administered concomitantly with routine infant and child vaccinations: results of two randomised trials. Lancet 2013;381:825-35.

7. Summary of product characteristics: Bexsero. London (UK): European Medicines Agency; 2013. Available: www.ema.europa .eu/docs/en_GB/document_library/EPAR_-_Product_Information /human/002333/WC500137881.pdf (accessed 2013 May 8).

8. Ramsay M. Current epidemiology of meningococcal disease in the UK and Europe. London (UK): Meningitis Research Foundation; 2011.

9. Findlow J, Holland A, Andrews N, et al. Comparison of phenotypically indistinguishable but geographically distinct Neisseria meningitidis Group B isolates in a serum bactericidal antibody assay. Clin Vaccine Immunol 2007;14:1451-7.

10. Jackson C, Lennon D, Wong S, et al. Antibody persistence following MeNZB vaccination of adults and children and response to a fourth dose in toddlers. Arch Dis Child 2011;96:744-51.

11. Clopper CJ, Pearson ES. The use of confidence or fiducial limits illustrated in the case of the binomial. Biometrika 1934;26:404-13.

12. Trotter CL, Andrews NJ, Kaczmarski EB, et al. Effectiveness of meningococcal serogroup $\mathrm{C}$ conjugate vaccine 4 years after introduction. Lancet 2004;364:365-7.

13. Holst J, Feiring B, Fuglesang JE, et al. Serum bactericidal activity correlates with the vaccine efficacy of outer membrane vesicle vaccines against Neisseria meningitidis serogroup B disease. Vaccine 2003;21:734-7.

14. Galloway Y, Stehr-Green P, McNicholas A, et al. Use of an observational cohort study to estimate the effectiveness of the New Zealand group B meningococcal vaccine in children aged under 5 years. Int J Epidemiol 2009;38:413-8.

15. Donnelly J, Medini D, Boccadifuoco G, et al. Qualitative and quantitative assessment of meningococcal antigens to evaluate the potential strain coverage of protein-based vaccines. Proc Natl Acad Sci U S A 2010;107:19490-5.

16. Donnelly J, Medini D, Giuliani MM, et al. Estimating the potential strain coverage in Europe of a multicomponent vaccine targeting serogroup B meningococci. Proceedings of the 11th meeting of the European Meningococcal Disease Society (EMGM); 2011 May 18-20; Ljubljana (Slovenia).

17. Smith H, Nissen M, Sloots T, et al. Estimating the potential strain coverage in Australia of a multicomponent vaccine target ing serogroup B meningococci. Melbourne (Australia): World Society for Pediatric Infectious Diseases; 2011.

18. Bettinger JA, Scheifele DW, Halperin SA, et al. Diversity of Canadian meningococcal serogroup B isolates and estimated coverage by an investigational meningococcal serogroup $\mathrm{B}$ vaccine (4CMenB). Vaccine 2013; Apr. 12 [Epub ahead of print].

19. Hong E, Giuliani MM, Deghmane AE, et al. Could the multicomponent meningococcal serogroup B vaccine (4CMenB) control Neisseria meningitidis capsular group $\mathrm{X}$ outbreaks in Africa? Vaccine 2013;31:1113-6.

Competing interests: Matthew Snape and Andrew Pollard have conducted clinical trials on behalf of the University of Oxford, sponsored by Novartis Vaccines and Diagnostics, Pfizer, GlaxoSmithKline and Sanofi-Pasteur MSD. Andrew Pollard and Matthew Snape do not receive personal payments from vaccine manufacturers; grants for support of educational activities are paid to an educational/administrative fund held by the Department of Paediatrics, University of Oxford. Matthew Snape and Tessa John have received assistance to attend scientific meetings from Novartis, Pfizer and GlaxoSmithKline. Matthew Snape has spent a period of secondment at Novartis. Andrew Pollard chairs the European Medicines Agency's Scientific Advisory Group on Vaccines and is a member of the UK Department of Health's Joint Committee on Vaccination and Immunisation Meningococcal Sub-committee. Huajun Wang, Peter Dull and Daniela Toneatto are employees of Novartis Vaccines and Diagnostics. Praveen Saroey, Hannah Robinson, Sarah Kelly, Nicoletta Gossger and Ly-Mee Yu have no competing interests to declare. 
Affiliations: Department of Paediatrics (Snape, Saroey, John, Robinson, Kelly, Gossger, Pollard), University of Oxford and NIHR (National Institute for Health Research) Oxford Biomedical Research Centre, Oxford, UK; Centre for Statistics in Medicine (Yu), University of Oxford, Oxford, UK; Novartis Vaccines and Diagnostics (Wang, Toneatto, Dull), Siena, Italy

Contributors: Peter Dull and Daniela Toneatto prepared the study protocol in collaboration with Matthew Snape, Andrew Pollard and Tessa John. The study organization, conduct and oversight was performed by Tessa John, Hannah Robinson, Sarah Kelly, Nicoletta Gossger, Matthew Snape and Andrew Pollard (chief investigator). Data analysis was performed by Huajun Wang and independently verified by Ly-Mee Yu. Interpretations were performed by Matthew Snape, Praveen Saroey, Peter Dull and Andrew Pollard. Matthew Snape and Praveen Saroey drafted the article, which all authors revised. Praveen Saroey prepared the figures. All authors approved the final version of the manuscript submitted for publication.

Funding: This study was funded and sponsored by Novartis Vaccines and Diagnostics, which provided the study vaccine, performed laboratory analysis of the sera and developed this protocol in collaboration with Andrew Pollard, Matthew Snape and Tessa John. Data were analyzed by Novartis Vaccines and Diagnostics and independently verified by Ly-Mee Yu. Editorial control over content was held by the investigators at the University of Oxford. Matthew Snape and Tessa John are supported by the NIHR (National Institute for Health Research) Oxford Biomedical Research Centre. The research was supported by the NIHR Oxford Biomedical Research Centre based at Oxford University Hospitals NHS (National Health Service) Trust and University of Oxford. The views expressed are those of the authors and not necessarily those of the National Health Service, the NIHR or the Department of Health. Andrew Pollard is a Jenner Institute investigator and a James Martin senior fellow.

Acknowledgments: The authors thank the study participants and their parents, without whom this study would not have been possible. The authors also thank the research nurses Jen Houlden and Aimee Field for their efforts in organizing and conducting study visits, Emma Plested for her outstanding administrative support and the staff of the Child Health Computer Departments in Oxfordshire, Buckinghamshire, Berkshire and Northamptonshire. 